\title{
MINLP optimization of steel frames
}

\author{
S. Kravanja \& U. Klanšek \\ University of Maribor, Faculty of Civil Engineering, Smetanova 17, 2000 \\ Maribor, Slovenia
}

\begin{abstract}
In this paper we deal with the topology and standard optimization of unbraced steel frames with rigid beam-to-column connections. The optimization has been performed by the Mixed-Integer Non-linear Programming (MINLP) approach. The MINLP performs a discrete topology and standard dimension optimization, while continuous parameters are simultaneously calculated inside the continuous space. As the discrete/continuous optimization problem of steel frames is non-convex and highly non-linear, the Modified Outer-Approximation/EqualityRelaxation (OA/ER) algorithm has been used for the optimization. Two practical examples with the results of the optimization are shown at the end of the paper.
\end{abstract}

\section{Introduction}

The paper presents the topology and standard dimension optimization of unbraced steel frames with rigid beam-to-column connections. The optimization of frames is performed by the Mixed-Integer Nonlinear Programming, MINLP. The MINLP is a combined discrete-continuous optimization technique. In this way, the MINLP performs the discrete topology (i.e. the number of columns and beams) and standard dimension optimization (i.e. standard cross-section sizes) simultaneously with the continuous optimization of parameters (e.g. internal forces, deflections, mass, costs, etc.).

The MINLP discrete/continuous optimization problems of frames are in most cases comprehensive, non-convex and highly non-linear. This optimization approach is proposed to be performed through three steps. The first one includes the generation of a mechanical superstructure of different topology and standard dimension alternatives, the second one involves the development of an MINLP model formulation and the last one consists of a solution for the defined MINLP optimization problem. 
The Modified Outer-Approximation/Equality-Relaxation algorithm [1], [2] and [3] is used to perform the optimization. The objective of the optimization is to minimize the mass of the structure. The finite element equations are defined as the equality constraints for the calculation of internal forces and deflections of the structure. Constraints for the dimensioning of steel members are determined in accordance with Eurocode 3 [4]. Beside the theoretical basics, an example of the optimization of a steel frame is presented at the end of the paper.

\section{Steel frames}

Steel frames, see Figure 1, are proposed to be analyzed under the combined effects of the self-weight of frame members, uniformly distributed variable load, concentrated variable load on each storey and an initial frame imperfection.

Second-order elastic structural analysis is performed by considering a geometric nonlinearity due to $\mathrm{P}-\delta$ and $\mathrm{P}-\Delta$ effects. While the $\mathrm{P}-\delta$ effect is associated with the influence of the axial force on the beam-column member flexure, the P- $\Delta$ effect denotes the influence of axial force acting through the relative sideways displacements of the element ends. In this paper, both effects are included in the nonlinear stiffness matrix of the individual frame member by usage of stability function approach. Chen and Lui [5] have comprehensively described this approach and have presented stability functions sii and sij. These stability functions are different for compressive and tensile axial forces. Since they give the indeterminate numerical solution when axial force is zero, simplified expressions for stability functions $S 1$ and $S 2$, introduced by Kim et al. [6], are rather used. The shear deformation effect is neglected considering the fact that only slender structural members are subjected to buckling for which shear deformation is insignificant.

Design/dimensioning of steel frames is performed in accordance with Eurocodes 3 for the conditions of both the ultimate limit and serviceability limit states. When the ultimate limit state of beam-column members is considered, the elements were checked for bending moment, vertical shear, shear buckling, interaction between bending, shear and axial force and interaction between bending and axial compression/buckling. The ultimate moment capacity is calculated by the elastic method. Since the second-order elastic global analysis is used, the in-plane buckling lengths of compression members are calculated considering the non-sway mode.

Considering the serviceability limit state, the vertical deflections of beams in the individual storey were calculated by the elastic method. The total deflections subjected to overall load $\delta_{\max }$ and the deflections subjected to the variable imposed load $\delta_{2}$ are calculated to be smaller than the limited maximum values: $\operatorname{span} / 250$ and span/300, respectively. The horizontal deflections are also checked for the individual storey and for the structure as a whole. Both types of horizontal deflections are checked for the recommended limits: the relative horizontal deflection of each storey should be smaller than each storey height $/ 300$ and the horizontal deflection of the top of the frame must be smaller than an overall frame height/500. 


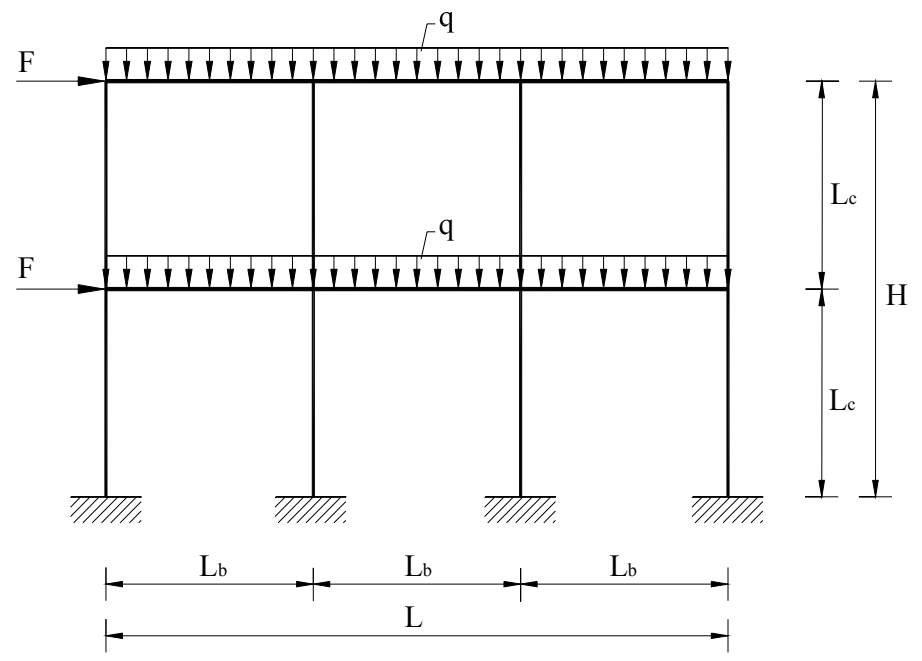

Figure 1: $\quad$ Unbraced steel frame.

\section{MINLP model formulation for mechanical superstructures}

The MINLP optimization approach requires the generation of an MINLP frame superstructure composed of various topology and standard dimension design alternatives that are all candidates for a feasible and optimal solution. It is assumed that a general nonconvex and nonlinear discrete/continuous optimization problem can be formulated as an MINLP problem (MINLP-G) in the form:

$$
\begin{gathered}
\min \quad z=\boldsymbol{c}^{T} \boldsymbol{y}+f(\boldsymbol{x}) \\
\text { s.t. } \quad \boldsymbol{h}(\boldsymbol{x})=\boldsymbol{0} \\
\boldsymbol{g}(\boldsymbol{x}) \leq \boldsymbol{0} \\
\boldsymbol{B} \boldsymbol{y}+\boldsymbol{C} \boldsymbol{x} \leq \boldsymbol{b} \\
\boldsymbol{x} \in X=\left\{\boldsymbol{x} \in R^{n}: \boldsymbol{x}^{\mathrm{LO}} \leq \boldsymbol{x} \leq \boldsymbol{x}^{\mathrm{UP}}\right\} \\
\boldsymbol{y} \in Y=\{0,1\}^{m}
\end{gathered}
$$

where $\boldsymbol{x}$ is a vector of continuous variables specified in the compact set $X$ and $\boldsymbol{y}$ is a vector of discrete, mostly binary $0-1$ variables. Functions $f(\boldsymbol{x}), \boldsymbol{h}(\boldsymbol{x})$ and $\boldsymbol{g}(\boldsymbol{x})$ are nonlinear functions involved in the objective function $z$, equality and inequality constraints, respectively. Finally, $\boldsymbol{B} \boldsymbol{y}+\boldsymbol{C} \boldsymbol{x} \leq \boldsymbol{b}$ represents a subset of mixed linear equality/inequality constraints.

The above general MINLP model formulation has been adapted for the optimization of mechanical superstructures (MINLP-SMS). The resulted formulation that is more specific, particularly in variables and constraints. It can also be used for the modelling of steel frames. It is given in the following form: 


$$
\begin{gathered}
\min \quad z=\boldsymbol{c}^{T} \boldsymbol{y}+f(\boldsymbol{x}) \\
\text { s.t. } \quad \boldsymbol{h}(\boldsymbol{x})=\boldsymbol{0} \\
\boldsymbol{g}(\boldsymbol{x}) \leq \boldsymbol{0} \\
\boldsymbol{A}(\boldsymbol{x}) \leq \boldsymbol{a} \\
\boldsymbol{E} \boldsymbol{y} \leq \boldsymbol{e} \\
\boldsymbol{D y}^{e}+\boldsymbol{R}(\boldsymbol{x}) \leq \boldsymbol{r} \\
\boldsymbol{K} \boldsymbol{y}^{e}+\boldsymbol{L}\left(\boldsymbol{d}^{c n}\right) \leq \boldsymbol{k} \\
\boldsymbol{P} \boldsymbol{y}+\boldsymbol{S}\left(\boldsymbol{d}^{s t}\right) \leq \boldsymbol{s} \\
\boldsymbol{x} \in X=\left\{\boldsymbol{x} \in R^{n}: \boldsymbol{x}^{\mathrm{LO}} \leq \boldsymbol{x} \leq \boldsymbol{x}^{\mathrm{UP}}\right\} \\
\boldsymbol{y} \in Y=\{0,1\}^{m}
\end{gathered}
$$

(MINLP-SMS)

In the model formulation, included are continuous variables $\boldsymbol{x}=\{\boldsymbol{d}, \boldsymbol{p}\}$ and discrete binary variables $\boldsymbol{y}=\left\{\boldsymbol{y}^{e}, \boldsymbol{y}^{s t}\right\}$. Continuous variables are partitioned into design variables $\boldsymbol{d}=\left\{\boldsymbol{d}^{c n}, \boldsymbol{d}^{\text {st }}\right\}$ and into performance (nondesign) variables $\boldsymbol{p}$, where subvectors $\boldsymbol{d}^{c n}$ and $\boldsymbol{d}^{\text {st }}$ stand for continuous and standard dimensions, respectively. Subvectors of binary variables $\boldsymbol{y}^{e}$ and $\boldsymbol{y}^{\text {st }}$ denote the potential existence of structural elements inside the superstructure (the topology determination) and the potential selection of standard dimension alternatives, respectively.

The mass or economical objective function $z$ involves fixed mass or cost charges in the linear term $\boldsymbol{c}^{\mathrm{T}} \boldsymbol{y}$ and dimension dependent mass or costs in the term $f(\boldsymbol{x})$.

Parameter nonlinear and linear constraints $\boldsymbol{h}(\boldsymbol{x})=\boldsymbol{0}, \boldsymbol{g}(\boldsymbol{x}) \leq \boldsymbol{0}$ and $\boldsymbol{A}(\boldsymbol{x}) \leq \boldsymbol{a}$ represent the rigorous system of the design, loading, stress, deflection, stability, etc. constraints known from the structural analysis.

Integer linear constraints $\boldsymbol{E} \boldsymbol{y} \leq \boldsymbol{e}$ are proposed to describe relations between binary variables.

Mixed linear constraints $\boldsymbol{D}^{e}+\boldsymbol{R}(\boldsymbol{x}) \leq \boldsymbol{r}$ restore interconnection relations between currently selected or existing structural elements (corresponding $y^{e}=1$ ) and cancel relations for currently disappearing or nonexistent elements (corresponding $y^{e}=0$ ).

Mixed linear constraints $\boldsymbol{K} \boldsymbol{y}^{e}+\boldsymbol{L}\left(\boldsymbol{d}^{c n}\right) \leq \boldsymbol{k}$ are proposed to define the continuous design variables for each existing structural element. The space is defined only when the corresponding structure element exists $\left(y^{e}=1\right)$, otherwise it is empty.

Mixed linear constraints $\boldsymbol{P} \boldsymbol{y}+\boldsymbol{S}\left(\boldsymbol{d}^{s t}\right) \leq \boldsymbol{s}$ define standard design variables $\boldsymbol{d}^{\mathrm{st}}$. Each standard dimension $d^{s t}$ is determined as a scalar product between its vector of standard dimension constants $\boldsymbol{q}$ and its vector of binary variables $\boldsymbol{y}^{\text {st }}$. Only one discrete value can be selected for each standard dimension since:

$$
d^{\mathrm{st}}=\sum_{i \in I} q_{i} y_{i}^{\mathrm{st}} \quad \sum_{i \in I} y_{i}^{\mathrm{st}}=1
$$




\section{Optimization}

The Modified Outer-Approximation/Equality-Relaxation (OA/ER) algorithm is used to perform the optimization. The OA/ER algorithm consists of solving an alternative sequence of Non-linear Programming optimization subproblems (NLP) and Mixed-Integer Linear Programming master problems (MILP). The former corresponds to the optimization of parameters for a frame structure with fixed topology and standard dimensions and yields an upper bound to the objective to be minimized. The latter involves a global approximation to the superstructure of alternatives in which a new topology and new standard sizes are identified so that its lower bound does not exceed the current best upper bound. The search is terminated when the predicted lower bound exceeds the upper bound.

The optimization is performed sequentially in two different phases in order to accelerate the convergence of the OA/ER algorithm. The optimization starts with the topology optimization of the frame, while standard dimensions are relaxed temporary into continuous parameters. In the case of the standard dimension optimization only, the optimization begins with the continuous NLP optimization of the frame. When the optimal topology (continuous parameters) is found, standard sizes of cross-sections are re-established and the standard dimension optimization of cross-sections is then continued until the optimal solution is found.

\section{Examples}

\subsection{Example 1: three-storey frame}

The first example shows the standard dimension optimization of the three-storey, three-bay plane steel frame (see Figure 2). The frame is subjected to the selfweight, to the uniformly distributed imposed load of $50 \mathrm{kN} / \mathrm{m}$ and to the concentrated imposed load of $10 \mathrm{kN}$ on each storey. The frame is considered as a laterally supported plane frame. The frame superstructure has been generated in which all possible structures are embedded by different standard sizes variation. The superstructure comprises 24 different standard hot rolled European wide flange HEA sections (from HEA 100 to HEA 1000) for each beam and column separately. The material used is steel S 355.

The optimization was performed by the MINLP optimization approach. The MINLP optimization model for steel frames was used. The task of the optimization was to find the optimal structure mass.

The optimization was carried out by a user-friendly version of the MINLP computer package MIPSYN, the successor of PROSYN [1] and TOP [7]. As an interface for mathematical modelling and data inputs/outputs GAMS (General Algebraic Modelling System), a high level language, was used [8]. The Modified OA/ER algorithm and the two-phased optimization were applied (a single MINLP), where GAMS/CONOPT2 (Generalized reduced-gradient method) [9] 
was used to solve NLP subproblems and GAMS/Cplex 7.0 (Branch and Bound) [10] was used to solve MILP master problems.

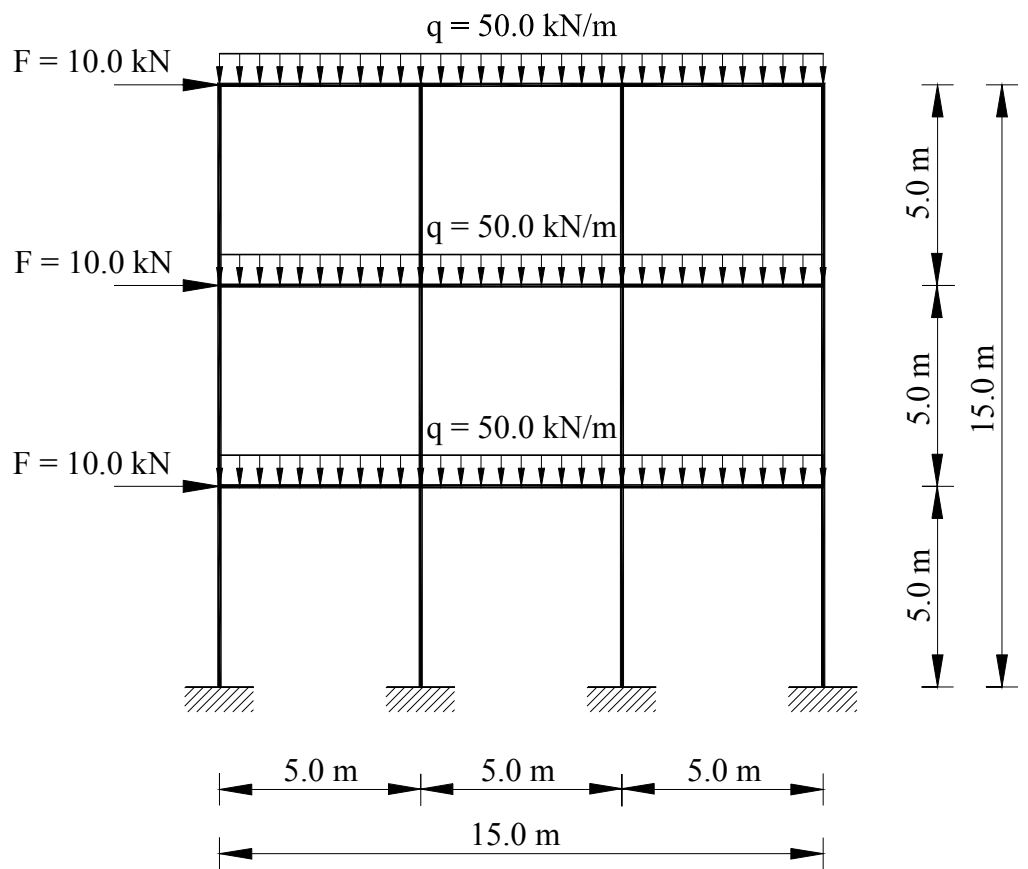

Figure 2: $\quad$ Three-storey, three-bay steel frame.

The optimization model of the frame contained 1572 (in)equality constraints, 1552 continuous and 357 binary variables (61 after the prescreening). The final optimal solution of $6214 \mathrm{~kg}$ was obtained in the $61^{\text {st }}$ main MINLP iteration (the subsequent feasible result was not so good). The optimal standard sizes were also obtained. Only 152 seconds of CPU time were spent on a $2 \mathrm{GHz}$ PC. The optimal structure of the frame is shown in Figure 3.

\subsection{Example 2: single-storey industrial building}

The second example presents the topology and standard dimension optimization of a single-storey industrial building. The building is 20 meters wide, 40 meters long and 6.5 meters high (see Figure 4). The structure is consisted from equal non-sway steel portal frames, which are mutually connected with the purlins.

The optimization was performed by the MINLP optimization approach. The task of the optimization was to find the optimal structure mass, the optimal topology (the optimal number of portal frames and purlins) and optimal standard cross-sectional sizes. 
Variable imposed loads $s=1.60 \mathrm{kN} / \mathrm{m}^{2}$ (snow) and $w=0.137 \mathrm{kN} / \mathrm{m}^{2}$ (wind) are defined as the uniformly distributed surface load in the model input data. Both, the horizontal concentrated load at the top of the columns (wind) and the vertical uniformly distributed line load on the beams (snow and wind) are calculated considering the intermediate distance between the portal frames. Internal forces are calculated by the elastic first-order analysis for non-sway frame mode. The dimensioning of steel members is performed in accordance with Eurocode 3 for the conditions of both ultimate limit state (ULS) and serviceability limit state (SLS).

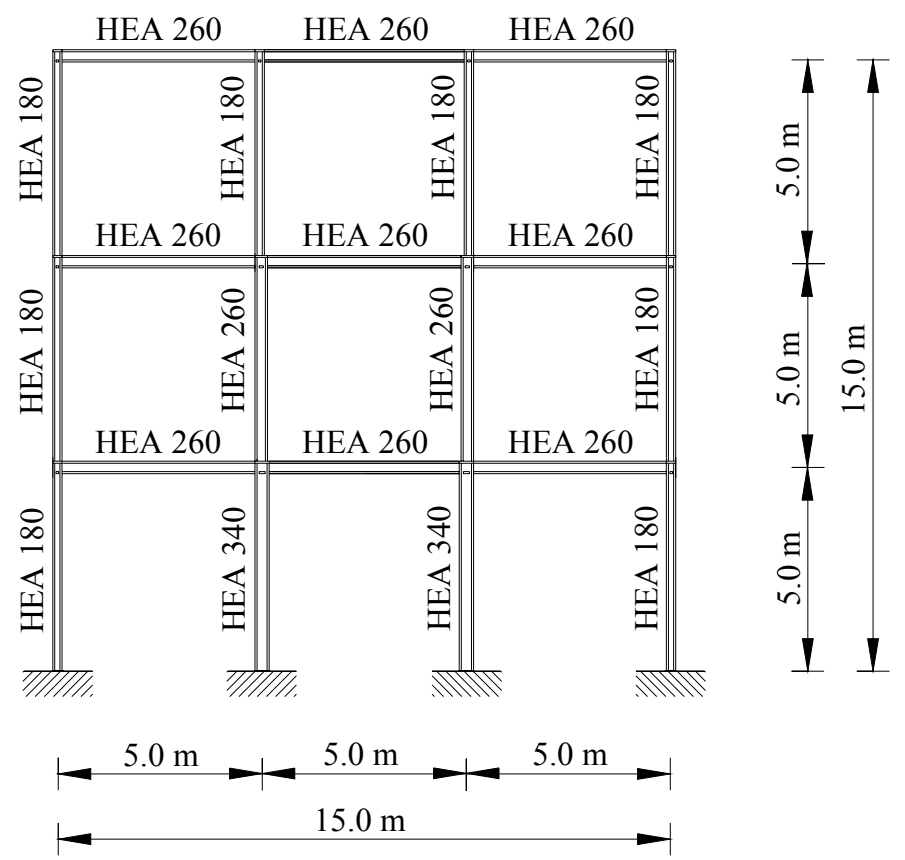

Figure 3: Optimum design of the frame.

The portal frame superstructure was generated in which all possible structures were embedded by 30 portal frame alternatives and 20 purlin alternatives. The superstructure also comprised 24 different standard hot rolled European wide flange I beams, i.e. HEA sections (from HEA 100 to HEA 1000) for each column, beam and purlin separately. The material used was steel S 355 .

The optimization model contained 124 (in)equality constraints, 111 continuous and 122 binary variables (55 after the prescreening). The final optimal solution of $62029 \mathrm{~kg}$ was obtained in the $3^{\text {rd }}$ main MINLP iteration. The optimal result includes the obtained optimal topology of 15 portal frames and 12 purlins (see Figure 4) as well as the calculated optimal standard sizes of columns, beams and purlins (see Figure 5). 


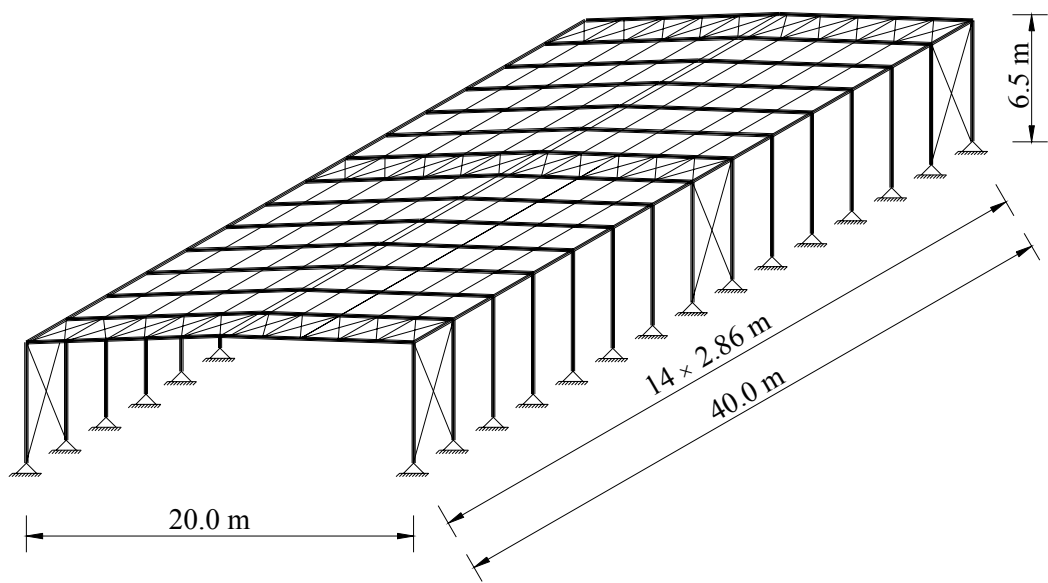

Figure 4: $\quad$ Optimal design of the single-storey industrial building.
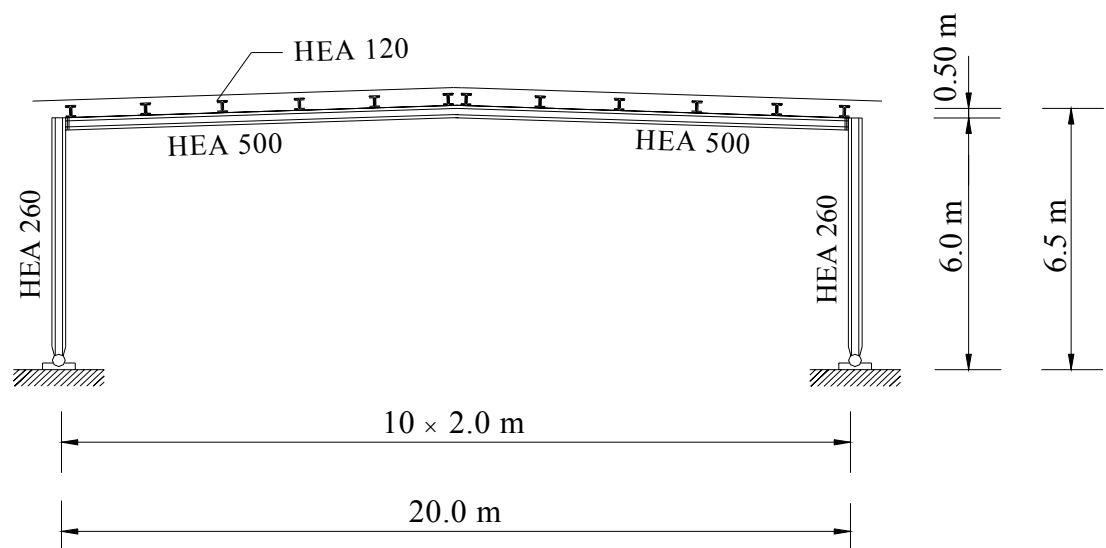

Figure 5: Optimal design of the portal frame.

\section{Conclusions}

This paper presents the topology and standard dimension optimization of steel frames with rigid beam-to-column connections. The optimization has been performed by the Mixed-Integer Non-linear Programming (MINLP) approach. The MINLP was found to be very successful optimization technique for solving the frame structures. 


\section{References}

[1] Kravanja, Z. and Grossmann, I.E., New Developments and Capabilities in PROSYN - An Automated Topology and Parameter Process Synthesizer, Computers chem. Eng., 18(11/12), pp. 1097-1114, 1994.

[2] Kravanja, S., Kravanja, Z. and Bedenik, B.S., The MINLP optimization approach to structural synthesis. Part I: A general view on simultaneous topology and parameter optimization, Int. J. Numer. Methods Eng., 43, pp. 263-292, 1998.

[3] Kravanja, S., Kravanja, Z. and Bedenik, B.S., The MINLP optimization approach to structural synthesis. Part II: Simultaneous topology, parameter and standard dimension optimization by the use of the Linked two-phase MINLP strategy, Int. J. Numer. Methods Eng., 43, pp. 293-328, 1998.

[4] Eurocode 3, Design of steel structures, European Committee for Standardization, 1992.

[5] Chen, W.F. and Lui, E.M., Structural Stability: Theory and Implementation, New York: Elsevier Science Publishing Co., Inc., 1987.

[6] Kim, S.E., Lee, J.S., Choi S.H. and Kim, C.S., Practical second-order inelastic analysis for steel frames subjected to distributed load, Eng. Struct., 26, pp. 51-61, 2004.

[7] Kravanja, S., Kravanja, Z., Bedenik, B.S. and Faith, S., Simultaneous Topology and Parameter Optimization of Mechanical Structures, Numerical Methods in Engineering '92, Proceedings of the First European Conference on Numerical Methods in Engineering, ed. Ch. Hirsch et al., pp. 487-495, Elsevier, Amsterdam, 1992.

[8] Brooke, A., Kendrick, D. and Meeraus, A., GAMS - A User's Guide, Scientific Press, Redwood City, CA, 1988.

[9] Drudd, A.S., CONOPT - A Large-Scale GRG Code, ORSA J. Comput., 6 (2), pp. 207-216, 1994.

[10] CPLEX User Notes, ILOG inc. 